\title{
D. D.
}
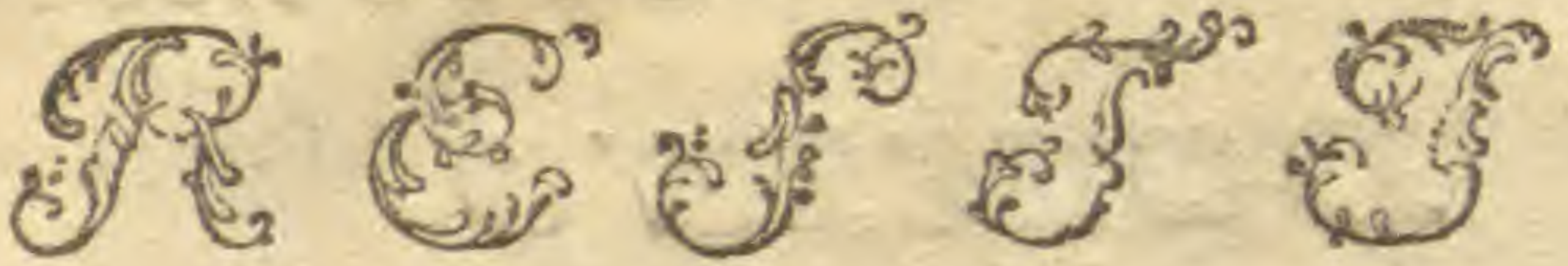

Q U E M

\section{9)}

\section{DISSERTATIONE BOTANICA,}

CONSENSU EXP. FAC. MED. UPSAL.



Equite Aurat, Reg Ord, de Vasa,

Medic. Doct, Pkopass. Med. et Botan. Reg. et Orn, Acad, Cesar. Nat. Curios, Reg. Seient, londrnens. Hoimiens, Societ, Scient, Upsat. Patr. Holm Berol, Nat. Scrut. Lundens, Hartem. Amstetd, Zeland. Nidrosiens, Haiens, Nat. Scrut. Medic. Edimburg. et Nat. Studios, Iard,

Mgmbro; nec non acas. Scient. Paris. Monsfeliens. Agricul. Paris, Florentin.et Batavin. Ind, Or. Correspond.

Tublico examini frubjicit,

$$
\begin{gathered}
\text { PETRUS LUNDMARK, } \\
\text { NERICIUS. }
\end{gathered}
$$

IN AUDIT, GUST, MAJ. D. XVII. MAJ. MDCCLXXXVIII,

H. A, M. S.

- U P SA L I F, LITTERIS DIRECTOR. JOH. EDMAN. 


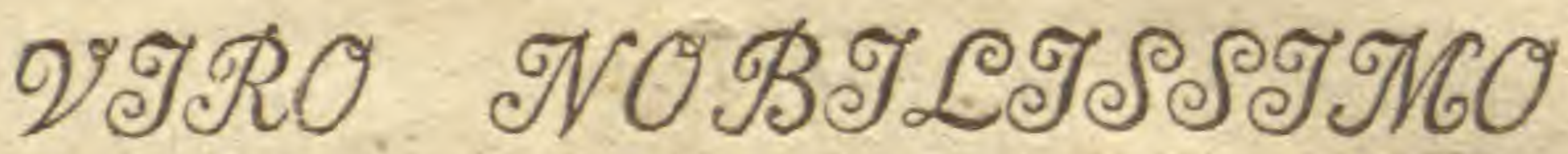

DD O . IVITIDO

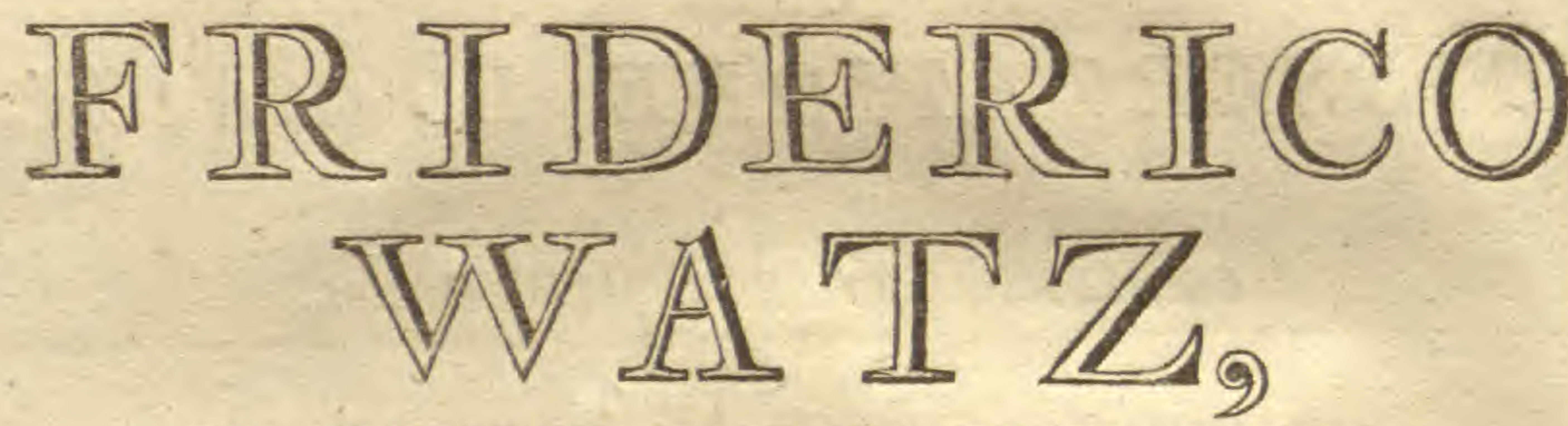

REI METALLICA PATRONO SPECTATISSIMO,

\section{PATRONO EETERNUM COLENDO}

Opufculum hocce Academicum ob magna in Se colla. lata beneficia, in tesseram grate anime dicatum, vo. luit, debuit 


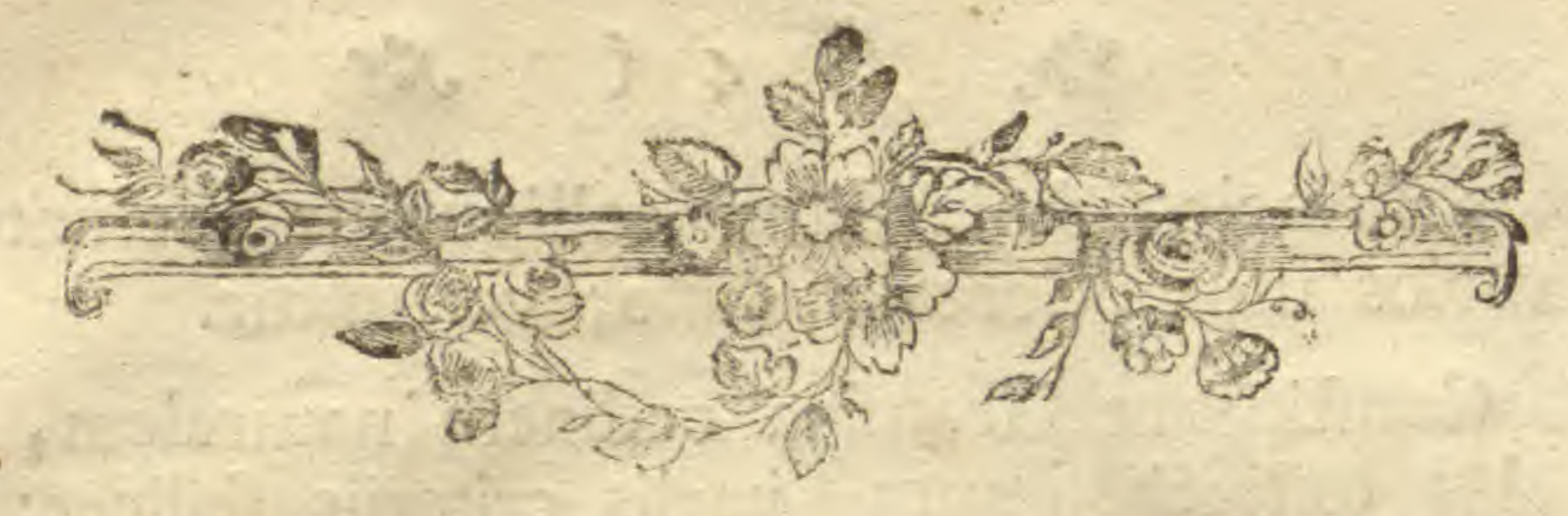

\section{S. I.}

75 ramina, vim roburque Regni vegetabilis con ftituentia, plerisque animalibus phytophagis edulia, ubique in hocce terraqueo Globo fpargere, disfeminare ac multiplicare Summo omnium rerum Creatori placuit; hæc, cetera inter vegetabilia, licet magis abjecta \& minus fuperba, tamen non parum utilia, omnium funt vulgatisfima, \& Plebejorum inftar, longe lateque diftributa, $a c$, ob floris partes minutas, difficile non minus examinanda quam digno. fcenda. Hac, inter quæ vix ullam reperies fpeciem venenatam, in regionibus calidioribus \& ficcioribus rarius quidem, at in frigidioribus dénfiffime tegunt \& virore fuo perenni exornant habitabilis noftri tel. luris fuperficiem, tegmenque virefcens non minus oculis noftris amcenum efficiunt, quam teneriores plantas alias $a b$ externis frigoris injuriis defendunt.

Sunt vera gramina vel perennia; vel annua, teneriora, quotannis florentia, femina ferentia ac fingulis annis disfeminanda. Quæ minora femina ferunt, Mammalibus \& avibus plerumque efcam præbent, dum illa, quæ majoribus fuperbiunt fpicis ac femini. 


\section{* 240}

bus, a Principe Animalium colliguntur, coluntur \& in varios ufus, Cerealia dicta, vertuntur.

Conftituunt Gramina ordinem naturalem, facile $\mathrm{ab}$ aliis diftinetisfimum, variis criteriis fcilicet:

Culmo fimplici, rarius ramolo, inani, articulato, raro frutefcénte.

Folits fimplicibus, vaginantibus, alternis, plerumque integris.

Floribus rarisfime coloratis, glumis valvulisque membranaceis, fæpisfime hermaphroditis, quandoque monoicis \& dioicis, nec non interdum polygamis.

Staminibus utplurimum tribus, raro fex, rarius uno vel duobus.

Seminibus nudis, rarisfime angiofpermis.

In hocce ordine naturali culmi \& folia Bettiis edulia funt, virore fuo perenni fuperficiem telluris exornant, domibus obtegendis, mattas funibusque conficiendis, inferviunt, nec non igni alendo materiam præbent. Semina farinofa \& glutinofa eximie nutriunt, homini imprimis in efcam cedunt, \& in hunc finem fere ubique coluntur.

\section{§. II.}

RESTronis genus, gramina inter, hoc primum feculo, nec multis abhinc annis detectum fuit in $\mathrm{C}_{2}$. pite bonæ fpei Africes, ejusque fpecies fenfim multiplicatz fuerunt. ElegIa quidem proprium ante conftituit genus; poftea vero huc relata fuit; fub no- 


\section{* ) $5($ )}

mine Reftionis Elegia; cum vero Calyx Elegia valde differat a Calyce Reftionis, confultius nos duximus, illud graminis genus iterum reftituere atque fub Elegix nomine diftinctum confervare. Aliud, \& quidem novum graminis genus, Reftioni proximum, ac quafi medium inter Reftionem \& Elegiam, WrLDENowia dietum ab hocce noftro diftingvit Calyx polyphyllus, corolla 6-petala \& fructus drupaceus. Recentiori quidem ævo Reftionis genus accuratius fuit defcriptum, ab 11l. LinNmo, Bergio \& imprimis a RоттвÓLlio, additis iconibus optimis; cum vero plurimas adhuc novas fpecies in promontorio bonæ fpei collegerit, illisque Mufæum Academiæ ditaverit Celeb. D. Præles, operam operatam me non facturum credidi, fi, \& ante notas \& hucusque incognitas in unum colleetas, magis in ufum Tuum, B. L. illuftratas defcriptasque ederem.

\section{III. \\ CHARACTER GENERICUS.}

Flores dioici, collęti intra ftrobilum.

Strobilus ovatus vel oblongus, multiflorus.

CAL. 6-glumis compresfus, fubæqualis, raro valde inæqualis, glaber, uniflorus. Glumæ exteriores obverlæ, naviculares; interiores lanceolatæ, tenuiores.

Corolla nulla, nifi glumæ tres interiores.

StaM. Filamenta tria.

Antbera oblongx. 


\title{
$\Rightarrow \quad) 6($
}

Pist. Germen fuperum.

Stylus I plerumque, rarius duplex, rarisfime triplex.

Stigma raro fimplex, fxpisfime duo, rariffme tria, plumofa.

Peric. Semen obfcurum.

Affinis nimis Schceno, fed femper dioicus \& habitu diverfo.

Variant nonnihil partes fruetificationis ut CaLYX, qui compresfus \& fubæqualis in cernuo, umbellato, ariftato, fpicigero $\hat{\delta}$, erecto $\hat{\jmath}$, acuminato $\delta$, parvifloro $\hat{\delta}$, argenteo $\hat{\delta}$, verticillari, digitato, dichotomo, triticeo, virgato, paniculato, tbannochorto; compresfus \& inæqualis in imbricato, fcariofo, Jpicigero ?, tetragono.

Strues, qui unicus in R. imbricato, trifloro, Spicigero, ariftato, fcariofo; duplex in verticillari, tri. ticeo; triplex in verticillari.

Præterea nonnullæ fpecies variant CaLYCE æquali \& inæquali, in diverfo $\int e x u$, ut fpicigerus of \& ${ }^{\circ}$.

Strlis \& Stigmatibus duobus vel tribus ut verticillaris.

\author{
§. IV. \\ DIVISIO SPECIERUM. \\ * culmo fimplici. \\ a culmo aphyllo.
}

imbricatus. x. R. culmo fimplici aphyllo, fpica oblonga compresfa. 
*)

vaginatus. 2. R. culmo fimplici aphyllo, fpicis alternis erectis, fquamis acuminatis.

arifatus. $\quad 3 \cdot R$. culmo fimplici aphyllo, fpicis terminalibus obovatis erectis, fquamis ariftatis.

cernuzs. 4. R. culmo fimplici aphyllo, fpicis turbinatis pendulis, fquamis obtufis cum acumine.

umbellatus. 5. R. culmo fimplici aphyllo, fpicis umbellatis ovatis, fquamis oblongis obtufis.

Spicigerus. 6. R. culmo fimplici aphyllo, fpicis oblongis hexagonis, fquamis lanceolatis apice patulis.

tectorum. 7. R. culmo fimplici aphyllo, fpicis racemofis fublecundis, fquamis fufcis nitidis.

ocuminatus. 8.R. culmo fimplici aphyllo, panicula erecta, fquamis ariftatis.

parviflorus. 9. R. culmo fimplici aphyllo, panicula erefta, fquamis rotundatis membra naceis.

erectus. so. R. culmo fimplici aphyllo, panicula erecta involucrata, pathis imbricatis lanceolatis.

argenteus, II. R, culmo fimplici aphyllo, panicula erecta, fquamis lanceolatis fcariofis. b. culmo foliofo.

Scariofus. 12. R. culmo fimplici foliofo, fpicarum fquamis lanceolatis fcariofis. 


\section{औ ) 8(}

thamnochortus. 13. R. culmo fimplici foliofo, panicula patenti, fquamis lanceolatis margine fcariofis.

fruticofus. 14. R. culmo fimplici foliofo, panicula compofita, fquamis fcariofis laceris.

fimplex. 15. R. culmo fimplici fubfoliofo, fpicis alternis fubagoregatis, fquamis ovatis. triflorus. 16. R. culmo fimplizi foliofo, fpicis alternis fesfilibus.

\section{* culmo ramofo. \\ a culmo aphyllo.}

tetragonus. I7. R. culmo ramisque tetragonis, fpicis alternis.

triticeus. 18. R. culmo dichotomo aphyllo erecto, ramis terétibus, (picis alternis.

glomeratus.19.R. culmo dichotomo aphyllo lavi, panicula glomerata.

incurvatus. 20.R. culmo dichoromo aphyllo ftrįto, fpicis imbricato - aggregatis.

digitatus. 2I. R. culmo dichotomo aphyllo ramis teretibus, fpicis ternis oblongis. verticillaris 22 . R. ramis verticillatis, panicula compofita. b. culmo foliofo.

Siopa. 23. R. culmo dichotomo foliofo, ramis compresfis, panicula fpicis glomeratis. virgatus. 24. R. culmo dichotomo foliofo, ramis compre fis, fpicis paniculatis pendulis. paniculatus.25. R. culmo dichotomo foliofo, ramis com. 


\section{* ) $9(2 \%$}

paniculatus.25. R. culmo dichotomo foliofo, ramis compresfis, fpicis fesfilibus alcernis ere tis. dicbotomus. 26. R. culmo dichotomo foliofo decumben. te, ramis teretibus, fpicis folitariis alternisque.

\section{§. V. \\ DESCRIPTIO SPECIERUM.}

Radix perennis.

Culmus fimplex vel ramofus, frpe dichotomus, aphyllus vel foliofus; femper articulatus, vaginatus ftipulis membranaceis; teres, rarius femiteres, rarisfime angulatus.

Rami fxpius dichotomi, rarius verticillati. Folia fetacea, dichotome ramofa.

I. R. Imbricatus: Culmus teres, fimplex, aphyllus, articulatus, erectus, bipedalis \& ultra.

Folua nulla; fed ftipulæ juxta articulos convolut:, vaginantes.

Spica fimplex, terminalis, ovato-oblonga, compresfa, brunnea, glabra, ereeta, pollicaris.

sqranne imbricatz apice patulo, oblongx, acutæ, concavæ, glabræ, imprimis verfus apicem brunner.

Calyx compresfus, inxqualis, 6-glumis: glu. mæ duæ anteriores naviculares, obverfæ, majores; quatuor interiores lanceolatx.

Siylus unicus.

Stigmata duo, clavata, plumofa.

Facile dignofcitur fpica terminali folitaria. B 


\section{$\Rightarrow \quad$ ) 10(}

2. R. Vaginatus: Culmus \& Stipule omnino ut in R. imbricato.

Spica alternx, fubfesfiles in rachide flexuofa, oblongx, erętr, tres vel quacuor.

Squance imbricata apice patulo, concava, ovatæ, acuminatæ, glabræ, brunnex, margine membranaceo pallidiore lacero.

Spicas ante explicationem florum tantum, nec flores vidi.

Aliqua hujus fimilitudo quidem eft cum Reftione diftachyo D. RotrtósLı; at revera diftinctus, ob fpicas plures \& fquamas fpicarum lato-ovatas, concavas, acuminatas, læves, laceras.

3. R. Aristatus: Culmus ut in R. cernuo.

Spica terminales, erectx; Iolitarix vel dux approximatæ, turbinatæ, usque quinque, oblongx.

Squame denfe imbricatæ, ovatæ, concavæ, fetaceo-ariftatæ, glabræ, brunnex.

th Calyx 6.glumis: glumæ xquales, obovatx feu inferne attenuatæ, acutæ, ferrugineæ, ung. viculares.

Filamenta capillaria, alba, longitudine corollæ. Antber ce lineares fufcx, flavo-ftriatæ.

o Stylus unicus, brevis.

Stigmata duo, plumofa, patentia.

4. R. Cernuus: Culmus fimplex, filiformis, articulatus, glaber, erectus, bipedalis \& ultra.

Folia nulla; fed in articulis vaginæ oblongæ, obtufi. 


\section{J ) II (}

Spice tres, quatuor vel quinque, pedunculatæ, turbinatæ, obtufæ, cernuæ, magnitu. dine pifi.

Pedunculi capillares.

Strobili Squama imbricatæ, rotundatæ, obtufre cum acumine, brunnex, glabræ.

Calyx fubrqualis, comprefus, 6-glumis: glumæ lanceolatæ, acutæ, glabræ.

Filamenta tria, brevifima.

Antbere lineares, flava.

Flores femineos non vidi.

5. R. Umbellatus: Culmus ut in R. cernuo, bipeda. lis \& ultra.

Spice tres vel plures, umbellatx umbella fimplici vel compofita, obovatæ, obtulæ, patentes, pifo majores.

Pedunculi capillares.

Squame fexfariam imbricatx, concavx, oblongæ, obtulæ, brunnex margine pallidiore, glabræ.

Calyx \& Stannina omnino ut in R. cernuo. Differt a R. cernuo, cui valde fimilis.

I:o Spicis pluribus, umbellatis, ovatis. 2:0 Sqvamis f́picarum oblongis, obtufis.

6. R. Spicigerus: Culmus fimplex, teres, fruticefces, aphyllus, articulatus, glaber, erectus, bipedalis \& vltra.

Folia nulla; fed in fingulo articulo ftipula vaginans, pollicaris.

d. Spice umbellatæ a medio fere culmo ad a. $\mathrm{B}_{2}$ picem, 


\section{औ) $) 12($}

picem; umbellæ fubpaniculatæ, patenti - cernu$æ$, plurimæ, oblongæ, fubcylindricæ, hexagonx, erectx, fubpollicares.

Squame fexfariam imbricatæ, apice patulo; lanceolatæ, acuminatæ, concavæ, fubcarinatæ, brunnex, glabræ.

Pedunculi trigoni, glabri, lati, flexi.

Calyx compresfus, inæqualis: glumæ binæ majores, obverfæ, naviculares, ovato-lanceolatæ, fquamis breviores; quatuor interiores ovatæ, minores.

Obf. Spica hujus fpeciei inftar amenti oblongi eft: Mas fpicis paniculatis, effufis, minoribus. Fernina f́picis racemofis majoribus.

Stylus unicus.

Stigrna fimplex, plumofum.

Filamenta brevisfima.

Antber a oblongæ, longitudine calycis.

o. Spica a medio culmo ad apicem racemofa, erectx, oblongx, obtufx, breves, glabræ, fubhexagonæ, craffitie fere digiti, ungviculares usque pollicares.

Squamse fexfariam imbricatæ, lato-Ianceolatæ apice acuto patulo, concavæ, fubcarinatæ, brunnex, glabræ.

Pedunculi trigoni, ftrieti, glabri.

Caly $x$ inæqualis, compresfus, concavus, 6glumis: glumæ dux exteriores naviculares, obverfx, majores; interiores quatuor lanceolatx, minores. 


\section{$*$ ) $13 .($ \%}

7. R. TeстоRum: Culmi e radice plures, fimplices, aphylli, filiformes, fubcompresfi, glabri, læves, erecti, articulati, tripedales vel ultra.

Vagine articulorum nigræ, acutæ, glabræ, decidux.

Spice fubpaniculatæ, adpreffæ, plurimæ in fummitate culmi.

Spicule pedicellatx, glomeratæ, fubfecundæ, trigonæ, acutæ.

Squama trifariam imbricatæ, concava, ovato-lanceolatx, acutæ, nitidæ, exteriores nigræ, interiores brunnex.

8. $R$, Acuminatus: Culmus ut in plurimis præcedentibus.

Spice fubpaniculata, erectx, ovatæ, bracteatæ.

Pedunculi ex articulo fingulo tres vel plures inæquales.

Bractee fub fingulo flore ovatæ, acumina: to - ariftatæ, glabræ, brunnex.

Calyx 6-glumis, æqualis: glumæ concavæ, ovatæ, obtufx, glabræ, minimæ.

Filamenta brevisfima.

Antberce ovatæ, fulcx.

Feminam non vidi.

9. R. Parviflorus: Culmus ut in præcedentibus plurimis.

Panicula terminales, aggregatæ.

$$
\text { B } 3
$$




\section{*}

Spice ovatx, obtulx, ereetx, ftrobiliformes; fquamæ rotundatæ, concavæ, margine membranacex.

Calyx 6-glumis, fubrqualis: glumæ ob. longx, interiores albidx, exteriores brunnex, minimx.

Filamenta brevisfima.

Antberce ovatæ, exfertæ, didymæ, ferrugineæ, flavo - Ariatæ.

Feminam non vidi.

10. R. ERectus: Culmas ut in R. cernuo \& plurimis præcedentibus.

Panicula alternæ, tres vel quatuor, ereetæ, effuix.

Bractea in bafi finguli pedunculi \& pedicelli compresfa, lanceolata, convoluta, acuta, glabra, parva.

£. Calyx 6-glumis: glumæ æquales, lanceolalatæ, glabræ.

Filnmenta breviffima.

Antbere oblongx.

Femineos flores non vidi.

11. R. ARgenteus: Culmus ut in plurimis præcedentibus.

Spica racemofo - paniculatx, feu e gemmis racemi plures inæquales, fpiculis fubfeifilibus, ovaris, erectis, ftrobiliformibus.

Squame imbricatæ, totæ fcariofæ, argenteo. nitentes, lanceolatæ, acuminatæ.

1. Calyx 6-glumis, æqualis: glumæ lanceolatæ, acuminatæ, concavæ, glabræ. 


\section{" ) 15 ( )}

Forsinam non vidi.

12. R. Scariosus: Culmus teres, fimplex, tenuiffime villofus, foliofus, fruticefcens, ereetus, pedalis \& ultra.

Folia e vaginis plura, dichotome ramofa; laciniæ paniculatæ, filiformi-capillaceæ, ftipulatæ ftipulis laceris argenteis.

Florum fpicæ in mare paniculatæ, effufx, oblongx; in femina racemofa \& fubfeffiles, ovatio.

Squame imbricatæ, lanceolatæ, totæ fcariofx carina oblcuriore, argenteæ, ungviculares.

Calyx compresfus, inæqualis, $6 \cdot$ glumis: glumæ duæ exteriores majores, naviculares, compreflx, obverfæ, acutæ, margine membranacex; interiores quatuor lanceolatæ.

obf. Calyx feminini foris duplo latior.

Stylys unicus.

Stigma fimplex, plumofum.

13. R. Thamnochortus: Culmus bafi decumbens, radicans, fquamatus, culmos plures edens teretes, glabros, erectos, foliofos, pedales \& ultra.

Folia per culmum totum fparfa, e vaginis alterna, dichotome ramofa laciniis fubulatis, ftipulata ftipulis argenteis, patentia.

Panicula terminalis, patens in mare (in femina fpicx racemofx videntur) e fpicis ovatis, ferrugineis.

Squame imbricatx, lanceolatæ, acuminatæ, ferrugineæ margine argenteo fcariofo, glabræ. 


\section{\#) $16($ (}

A. Calyx 6-glumis, parum compresfus, fubæqualis: glumx lanceolatx; exteriores dux paulo longiores, obverfx.

f. Calyx compresfus, latior.

ॠ4. R. Fruticosus: Culmus bafi fquamofus, teres, vaginatus, truticefcens, glaber, erectus, fimplex feu fuperne paniculatus, tripedalis \& ultra.

Vagine alternx, fetaceo-acuminatz, laceræ, fulcæ.

Folia heic, ut in R. thamnochorto, fed magis protracta \& elongata.

Flores a medio culmo ad apicem paniculati.

Pedunculi communes compreffi, glabri, inxquales.

Bractere fingulos flores veftientes acuminatæ, fcariofx, laceræ.

Flores explicatos non vidi, fic ut partes fru. Etificationis examinare non licuerit.

Differt a R. chamnochorto, quod ftrobilus heic nullus.

15. R. Simplex: Culmi e radice plurimi, filiformes, articulati, fimplices, tenuiffime ftriati, erecti, bipedales.

Foliola in culmo rarifima, fparfa.

Spica alternæ, folitariæ \& aggregatæ, breviter pedunculatx.

Squame ovatæ, concavæ, fub apice carinatæ, acuminatæ, glabræ.

16. R. TrIflorus : culmi e radice cælpitola plures, filiformes, lati, glabri, fimplices, ereeti, peda. les \& paulo ultra. 


\section{$\rightarrow \quad) 17($ )}

Folia plurima radicalia, pauciora in culmo, filiformia vel capillaria, articulata, vaginata, dichotome fed rariffime divifa, ereeta, culmum fubæquantia.

Spice terminales, feffiles, alternæ; plerumque tres, rarius plures, ovatæ.

Squame imbricatæ, acutæ, brunnex margine membranaceo.

Flores non vidi.

Diverfus certe a R. fimplici Forferi.

17. R. Tetragonus: Culrnus fruticefcens, tetragonus angulis acutis, aphyllus, glaber, erectus, bipedalis \& ultra.

Rami alterni, pauci, floriferi, aphylli, ereeti.

Vagine articulorum oblongx, acutæ, membranacex.

Spice in ramis \& ramulis alternæ, feffiles vel terminales, ovatæ, acutæ.

Squame imbricatæ, ovatæ, acutæ, glabræ, brunnex, margine pallidiore.

Calyx 6-glumis: glumæ lanceolazx, exteriores duæ obverfæ, majores lanceolatæ, carina ciliatx; interiores tenuiffim $x$, minores.

Filànenta breviffima.

Antberce oblongx.

18. R. Triticeus: Culmus trichotomus \& dichotomis, erectus teres, glaber, papillofus punctis albis parum fcabris, bipedalis \& ultra.

Rami filiformes, culmo fimiles.

Vagınce ramificationum ovatæ, obtufæ cum acumine, fulcx. 


\section{* ) $18($ )}

Spica alternæ, circiter quinque, ovatæ. Squame imbricatæ, ovatæ, concavæ, acutæ, unifloræ, glabræ.

Calyx fubxqualis, 6-glumis: glumx exteriores parum majores obverfæ, lanceolatæ; interiores paulo anguftiores, albidx.

Styli duo, fetacei, villofi, longitudine calycis. Filamenta tria, capillaria, albida, longit.calycis. Antbera oblongre, didymx.

19. R. Glomeratus: Culmus teres, lævis, dichotomus, ereetus, pedalis \& ultra.

Rami fimiles.

Flores terminales, paniculati fpicis glomeratis. Squanice ovatæ, acuminatæ, flavelcentes, fufco-irroratæ, glabræ.

Flo es non vidi.

20. R. Incurvatus: Culmus teres, frutefcens, dichotomus, ftriatus, glaber, bipedalis \& ultra. Rami alterni, fimiles, recurvi.

Vagina ovatx, acuminatx. Spica in ultimis ramis aggregatæ, feffiles, imbricatæ.

Squance ovatæ, acuminatæ, glabræ. Flores non vidi.

21. R. Digitatus: Culmus dichotomus, erętus, glaber, pedalis \& ultra.

Rawi $i$ compreffi, geniculati, dichotomi, glabri, faftigiati.

Vagince ramificationum lanceolatæ, glabræ. Spica circiter tres, terminales, oblongx, ferrugineæ, ungviculares. 


\section{$\Rightarrow$ ) 19 (}

Squarnce laxe imbricatæ, fubinflato-concavx, ovata.

1.. Calyx nullus nifi fquamulæ minutiffimx, tenuiffimæ, albidæ intra fquamam fingulam f́picx. Filamenta tria, brevia.

Antberce ovatx.

22. R. Verticillanis: Culmus frutefcens, teres, articulatus, lavis, glaber, erectus, craffitie calami, orgyalis.

Rami verticillati, plurimi, filiformes fimplices \& dichotomi articulati, glabri, ereeti, internodiis pluries longiores.

Fola nulla, fed vaginæ fub verticillis \& in articulis, feffiles, ovatæ, coriaceæ, glabræ, decidua, fenfim minores in ramis.

Panicula compofita, patens, fpicis in ultimis ramificationib us flexuofis feffilibus ovatisminutis.

Squama ovatz, obtufx, margine membranacex, glabræ, ferruginex.

Calyx 6-glumis, xqualis: glumæ lanceolatæ. Filamenta tria, brevifima.

Antbere ovatz.

Styli duo, \& tres. Stigmata duo vel tria villofa, fetacea.

23. R. Scopa: Culmus uc in R. paniculato.

Rami terminati in folia fetacea, fufci.

Folia in ramis fparfa eosque terminantia, comprefla, fetacea, erecta, fufca, faftigiata.

Flores in ramulis paniculatis glomerati.

Squame ovatx, concave, acuminato-arifta- 


\section{औ ) $20($ )}

tx, flavefcentes, fufco irroratæ, imbricatæ, interiores fenfim minores.

Partes floris \& fruct,ficationis non vidi,

24. R. Virgatus: Culmus frutefcens, glaber, ereetus, trichotomus \& dichotomus, bipedalis, \& ultra. Rami compreffi vel femiteretes, dichotomi, terminati in folia, ereeti, faftigiati.

Vaginie ramorum \& ram lorum oblongx, acuminatæ.

Folia in ultimis ramulis, filiformi-fetacea.

Florum ícic paniculatæ panicula patenti , acutæ.

Squame imbricatæ, ovatæ, obtufx, brunnex, glabræ, laceræ.

Calyx 6 -glumis : gluma exteriores obver$\mathrm{f} \mathfrak{x}$, lanceolatæ, parum majores; interiores quatuor tenuiffimx, pellucidær.

Filamenta breviffima.

Antber o ovatx, brunnex, luteo-ftriatx.

25. R. Paniculatus: Culmus ut in R. Virgato. Rani dichotomi, compreffi feu femiteretes, virgato-paniculati, glabri.

Folia in ramis dichotome ramofa, fetacea, curvata, vaginis membranaceis bafi cincta.

Spice in ramorum apicibus dichotomis fes. files \& alternx, ovatx, erectx.

Squand imbricatæ, ovatæ, vix acutæ, concavæ, ferrugineæ margine albo membranaceo. 1. Calyx 6-glumis: glumæ ut in R. Virgato: Differt a $R$. virgato: foliis capillaceis curvatis. Spicis feffilibus alternis ereetis, Jquamis obtufis. 26. $R$. 
26. R. Bicнотомus: Culmus teres, decumbens, flexuolo-erectus, glaber, papillofus, dichotomus. Rami filiformes, dichotomi, fubfaftigiati, papillofi, culmo fimiles.

Vagine ramificationum ovatæ, acutæ.

Folia in ramis fparfa, dichotome ramofa, filiformi-fubulata, curvata.

Spica in apicibus ramulorum alternæ, folitariæ usque quinque, oblongæ, glabræ, erectæ.

Squamia imbricatæ, oblongx, acutæ, concavæ. 1. Calyx 6-glumis: glumæ fubrquales, lanceolatæ; exteriores duæ obverfx, parum majores; interiores tenuiores.

Filamenta tria, calyce breviora.

Antberce ovata, fufcr.

Variat: Culmo magis vel minus decumbente. Ramis filiformibus vel capillaribus. Spicis folitariis vel pluribus alternis.

\section{§. VI: \\ SYNONYMA.}

R. cernuus, Linn. Syft. Veg. XIV. p. 882 . tectorum. Chondropetalum deuftum. Rotrb. p. Io. tab. 3. fig. 2. Reftio tectorum. Linn Supl: Syft. p. 425. Syft. Veg. XIX. p. 882 . acuminatus. Chondropetalum nudum. Kottb. Gram. p. 11. tab. 3. fig. 32 .

scariofus. Thamnochortus fruticolus. Berg. Plank. capenf. p. 353 . tab. 5. f. 8 . thamnocbortus. Reftio dichotomus. Rottb. Gram. p. 2. tob. I. fig. I. \&. C 3 


\section{$-2)=2(2$}

tîffoarus. Rottb. Gram. p. 3. tab. 2. fig. 2. S. triticetus. Rottb. Gram. p. 7. tab. 3. fig. 1. 1. . . verticillavis. Linn. Syft. Veg. XIV. p. 881. virgatus. Rottb. Gram. p. 5. tab. 1. fig. 2. \&. paniculatus. Rottb. Gram. p. 4. tab. 2. fig. 3. Linn. Syft. Veg. XIV. p. 88I.

dicbotomus. Schcenus capenfis. Linn. Spec. Pl. p. 64. Reftio dichotomus. Linn. Syft. Veg. XIV.p. 88I. Reftio vimineus. Rottb. Gram.p. 4. tab. 2. fig. I. Incolis Europais: Beefem riet. Lat. gramen fcopd §. VII.

Omnes in promontorio bonx fpei, (unica [pecie, R. fimplici, excepta, a Pr. Forstero inventa in nova Zelandia); crefcunt fpecies Reftionis, vulgatiffima in dunis, imprimis copiofe in campo illo arenofo vaftisfimo, qui littora maris a proxima ferie montium transverfa difcernit. Nonntllix quoque fumma ca: cumina montium inhabitare amant. Sic crefcit.

cernuts $\left.\begin{array}{l}\text { fcariofus } \\ \text { dicbotomus }\end{array}\right\}$

triticeus
tectorum

digitntits firi, montibus Hott. flolland.

verticillaris juxta margines rivulorum infra winterhok in Roodé fand, in Artaquas Kloof \& alibi.

$$
\begin{aligned}
& \text { §. VIII. } \\
& \text { U S U S. }
\end{aligned}
$$

Arenofa loca tegunt plurimæ fpecies frutefcentes \& animalibus refugia conciliant.

R. teftorum domibus obtegendis optime infervit, a vehementiflimis regionis ventibus \& procellis non movendus. Culmi rigidi, frutefcentes Pecoribus non appetuntur. 
